\title{
Traditional Chinese Medicine and COVID-19
}

\author{
Di Shen* \\ Department of Pharmacy, Wenshan Prefecture Dermatology Prevention and Treatment Center, China
}

*Corresponding author: Di Shen, Department of Pharmacy, Wenshan Prefecture Dermatology Prevention and Treatment Center, East of Xinping Road, Wenshan City, Wenshan Zhuang and Miao Autonomous Prefecture, Yunnan Province, China.

To Cite This Article: Di Shen. Traditional Chinese Medicine and COVID-19. Am J Biomed Sci \& Res. 2021 - 13(6). AJBSR.MS.ID.001932. DOI: 10.34297/AJBSR.2021.13.001932.

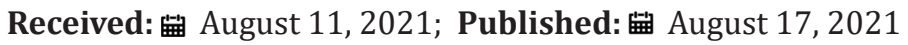

\section{Introduction}

As of August 5, 2021, WHO's official website statistics show that the cumulative number of confirmed cases of Coronavirus Disease 2019 (COVID-19) worldwide has exceeded 200 million. Although countries around the world are actively preventing its spread and make great efforts to treat patients with new coronary pneumonia, more than 4 million deaths have occurred.

The pathogen responsible for COVID-19 is Severe Acute Respiratory Syndrome Coronavirus 2 (SARS-CoV-2) [1]. Studies have shown that SARS-CoV-2 is a positive RNA virus with a wide natural host range, which could affect multiple system systemic functions in the human body [2]. Existing data have shown that COVID-19 is mainly manifested by fever, dry cough, as well as fatigue, etc. Subsequently, most severe patients developed dyspnea after 1 week, which rapidly progressed to acute respiratory distress syndrome, septic shock, refractory metabolic acidosis, and multiple organ failure [1,2].

Traditional Chinese medicine (TCM) is a therapeutic agent which was clinically applied under the guidance of traditional Chinese medicine theory. Based on the experience in the prevention and treatment of the SARS epidemic, China has taken extensive active measures to respond to the epidemic and issued guidelines for the diagnosis and treatment of COVID-19 patients, in which Chinese medicine treatment principles are recommended [3].

To date, more than $85 \%$ of SARS-CoV- 2 infected patients in China have received TCM treatment [4]. Many retrospective studies have shown that TCM can significantly alleviate the clinical symptoms of COVID-19. In March 2020, the Information Office of the State Council of China issued a report stating that approximately $91.5 \%$ of 7,4187 COVID-19 patients received TCM treatment and clinical efficacy observations showed that the total effective rate of TCM has reached more than $90 \%$ [3].

Based on the concrete clinical efficacy, TCM such as Qingfei Paidu Recipe, Xuanfei Baidu Recipe, Fossil Baidu Recipe, and Lianhua Qingwen Capsule have significant therapeutic effects on COVID-19 [5]. TMC has played a positive role in preventing COVID-19 patients from turning from mild to critical, blocking the deterioration of the disease thus greatly reducing the mortality rate. Taking Qingfei Paidu Recipe as an example, further studies have shown that it could ameliorate the symptoms caused by the disorder of ACE2 receptor expression in SARS-CoV-2 infection [4-6]. The multiple targets of Qingfei Paidu Recipe could inhibit activated cytokines, reduce excessive immune response, and eliminate inflammation $[5,6]$.

In China, the most frequently recommended TCM for COVID-19 treatment is Lianhua Qingwen Capsules. On April 12, 2021, Lianhua Qingwen Capsule was approved by the China National Medical Products Administration to increase the indications for COVID-19, which was clearly used for the treatment of "COVID-19 mild and ordinary" [7]. Studies have shown that Lianhua Qingwen Capsules can significantly inhibit the replication of SARS-CoV-2 in cells, significantly reduce the expression of viral particles in cells, and apparently inhibit the inflammatory factor TNF-a, IL-6, MCP-1 and IP-10 genes are overexpressed caused by SARS-CoV-2 infection of cells $[5,7]$. The therapeutic effects of TCM on COVID-19 have fully reflected its different pharmaceutical properties compared with western medicine. Modern pharmacological researches have found that TCM has multiple pharmacological effects, which can simultaneously strengthen the functions of various systems of the body at the same time [6]. This may be the maximum difference between TCM and western medicine. 
Although tremendous amount of data have shown that TCM is effective in treating patients with COVID-19, most patients also received other medical treatments, including antiviral drugs, nutritional support, and symptomatic support systems, etc. However, due to the suddenness of the COVID-19 outbreak and the urgency of TCM in combination with other treatment methods, long-term research on the effectiveness and safety of TCM needs to be further improved. Currently, COVID-19 vaccine is being actively pursued in many countries, but owing to insufficient vaccine production, uneven vaccine distribution, and vaccination contraindications, many people cannot be vaccinated in time [8] The popularization of TCM and the combined application with other treatments may make a greater contribution to the treatment of COVID-19.

\section{References}

1. Maciej M, Kowalik, Piotr Trzonkowski, Magdalena Lasinska-Kowara, Andrzej Mital, et al. (2020) COVID-19 - Toward a comprehensive understanding of the disease. Cardiology Journal 27(2) : 99-114.

2. Dhama K, Khan S, Tiwari R, Sircar S, Bhat S, et al. (2020) Coronavirus disease 2019 - COVID-19. Clin Microbiol Rev 33(4) : e00028-20.
3. Yuxi Li, Juan Li, Dongling Zhong, Yue Zhang, Yonggang Zhang, et al. (2020) Clinical practice guidelines and experts' consensuses of traditional Chinese herbal medicine for novel coronavirus (COVID-19): protocol of a systematic review. Systematic Reviews 9(1): 170.

4. Yang Yang, Md Sahidul Islam, Jin Wang, Yuan Li, Xin Chen (2020) Traditional Chinese Medicine in the Treatment of Patients Infected with 2019-New Coronavirus (SARS-CoV-2): A Review and Perspective 16(10): 1708-1717.

5. Xiaoqi Pana, Lan Dongb, Lian Yanga, Dayi Chena, Cheng Peng (2020) Potential drugs for the treatment of the novel coronavirus pneumonia (COVID-19) in China. Virus Res 286: 198057.

6. Wei Ren, Pan Liang, Yue Ma, Qin Sun, Qingrong Pu, et al. (2021) Research progress of traditional Chinese medicine against COVID-19. Biomed Pharmacother 137: 111310.

7. Chengyuan Liang, Nan Hui, Yuzhi Liu, Guaiping Qiao, Juan Li, et al. (2021) Insights into forsythia honeysuckle (Lianhuaqingwen) capsules: A Chinese herbal medicine repurposed for COVID-19 pandemic. Phytomedicine Plus 1(2): 2667-0313.

8. Olivier J Wouters, Kenneth C Shadlen, Maximilian Salcher-Konrad, Andrew J Pollard, Yot Teerawattananon, et al. (2021) Challenges in ensuring global access to COVID-19 vaccines: production, affordability, allocation, and deployment. Lancet 397(10278): 1023-1034. 